\title{
ESTUDO DA PURIFICAÇÃo DE BIOGÁS PARA USO ENERGÉTICO PELA ABSORÇÃO $\mathrm{DE}_{2} \mathrm{~S}$ E $\mathrm{CO}_{2}$
}

\author{
D. C. SCHIAVON MAIA ${ }^{1 *}$, R. R. NIKLEVICZ ${ }^{2}$, E. EYNG ${ }^{2}$, L. M. FRARE ${ }^{2}$, M. L. GIMENES ${ }^{1}$ e \\ N. C. PEREIRA ${ }^{1}$ \\ ${ }^{1}$ Universidade Estadual de Maringá, Departamento de Engenharia Química \\ ${ }^{2}$ Universidade Tecnológica Federal do Paraná, Departamento de Engenharia Ambiental \\ *e-mail: djeine.cr@ig.com.br
}

\begin{abstract}
RESUMO
$\mathrm{O}$ biogás contém pequenas quantidades de sulfeto de hidrogênio $\left(\mathrm{H}_{2} \mathrm{~S}\right)$, gás altamente tóxico e corrosivo. A combustão do $\mathrm{H}_{2} \mathrm{~S}$ junto com o biogás resulta na produção de dióxido de enxofre $\left(\mathrm{SO}_{2}\right)$ que causa severos problemas ambientais. Portanto, a dessulfurização deste gás é um pré-requisito para seu uso como fonte de energia. Além disto, para a utilização energética, o dióxido de carbono $\left(\mathrm{CO}_{2}\right)$ presente no biogás é considerado como inerte. O processo de purificação utilizado no presente trabalho é baseado na absorção química do $\mathrm{H}_{2} \mathrm{~S}$ por meio de uma reação de oxirredução promovida por Fe/EDTA em solução aquosa. Neste processo o enxofre obtido a partir do $\mathrm{H}_{2} \mathrm{~S}$ é oxidado à sua forma elementar mais estável, de fácil remoção. Além disto, promove-se a regeneração do Fe/EDTA em condições de temperatura e pressão ambientes pela a injeção de ar atmosférico. Embora o Fe/EDTA seja seletivo ao $\mathrm{H}_{2} \mathrm{~S}$, ocorre também a absorção física de dióxido de carbono na solução comercial por essa ser aquosa. Para avaliar a influência da remoção de $\mathrm{H}_{2} \mathrm{~S}$ e $\mathrm{CO}_{2}$ do biogás foram variadas a concentração de Fe/EDTA na solução e a relação entre a vazão de líquido e gás (L/G) por meio de um planejamento experimental utilizando a metodologia do Delineamento Composto Central Rotacional (DCCR). A partir da análise dos resultados obtidos, podese comprovar que a eficiência de remoção de $\mathrm{H}_{2} \mathrm{~S}$ é influenciada, significativamente pelas duas variáveis investigadas e a remoção do $\mathrm{CO}_{2}$ é unicamente dependente da relação L/G.
\end{abstract}

\section{INTRODUÇÃO}

A grande participação das fontes não renováveis na oferta mundial de energia coloca a sociedade diante de um desafio: a busca por fontes alternativas. Isso não pode demorar a ocorrer, sob o risco de o mundo, literalmente, entrar em colapso, pelo menos se for mantida a atual matriz energética, na qual o petróleo tem uma importância vital (MAGALHÃES et al., 2004).

O biogás é produzido por via anaeróbia da digestão de materiais orgânicos, tais como biomassa, lixo biodegradável, esgotos ou resíduos sólidos urbanos (SIGOT et al., 2014). Devido ao seu alto teor de metano, o biogás pode ser utilizado como um combustível para gerar eletricidade (KÁRÁSZOVÁ et al., 2012).

Frequentemente, as plantas de biogás são capazes de atingir sustentabilidade energética, utilizando o gás que produzem em suas próprias instalações (NUTIU, 2014).

O biogás consiste, principalmente, de $\mathrm{CH}_{4}$ e $\mathrm{CO}_{2}$. Outros componentes em menores quantidades podem estar presentes no biogás, 
como o vapor de água $\left(\mathrm{H}_{2} \mathrm{O}\right)$, sulfeto de hidrogênio $\left(\mathrm{H}_{2} \mathrm{~S}\right)$, amônia $\left(\mathrm{NH}_{3}\right)$, oxigênio $\left(\mathrm{O}_{2}\right)$, monóxido de carbono $(\mathrm{CO})$ e nitrogênio $\left(\mathrm{N}_{2}\right)$ (RYCKEBOSCH, DROUILLON e VERVAEREN, 2011). $\mathrm{O} \mathrm{CO}_{2}$ atua como um diluente no biogás (HINTON e STONE, 2014) e a remoção extra, mesmo que parcial, do dióxido de carbono aumenta, significativamente, seu poder calorífico. A presença do $\mathrm{H}_{2} \mathrm{~S}$ é extremamente indesejável devido ao seu efeito corrosivo nos queimadores e à formação de $\mathrm{SO}_{2}$ decorrente do processo de combustão que causa severos problemas ambientais, como a chuva ácida. Portanto, a dessulfurização do biogás é um pré-requisito para o seu uso como fonte de energia (MAAT, HOGENDOORNB e VERSTEEG, 2005).

A purificação do biogás tem atraído uma grande atenção da comunidade científica (KÁRÁSZOVÁ et al., 2012). $\mathrm{O} \mathrm{H}_{2} \mathrm{~S}$ pode ser removido de correntes gasosas pelo contato com soluções aquosas de Fe/EDTA. Segundo Wubs e Beenackers (1993), a absorção de $\mathrm{H}_{2} \mathrm{~S}$ é representada pelas Equações 1 e 2 .

$$
\begin{aligned}
& \mathrm{H}_{2} \mathrm{~S}_{(g)} \rightarrow \mathrm{H}_{2} \mathrm{~S}_{(a q)} \\
& \mathrm{H}_{2} \mathrm{~S}_{(a q)}+2 \mathrm{Fe}^{+3} / \mathrm{EDTA}_{(a q)} \rightarrow S \downarrow+2 \mathrm{H}^{+} \\
& +2 \mathrm{Fe}^{+2} / \mathrm{EDTA}_{(a q)}
\end{aligned}
$$

$\mathrm{O}$ composto $\mathrm{Fe}^{+2}$ /EDTA é regenerado para a forma férrica pela oxidação da solução com o oxigênio, conforme as Equações 3 e 4.

$$
\begin{aligned}
& \mathrm{O}_{2(g)} \rightarrow \mathrm{O}_{2(a q)} \\
& \mathrm{O}_{2(a q)}+4 \mathrm{Fe}^{+2} / \mathrm{EDTA}_{(a q)}+2 \mathrm{H}_{2} \mathrm{O} \rightarrow \\
& 2 \mathrm{Fe}^{+3} / \mathrm{EDTA}_{(a q)}+2 \mathrm{OH}_{(a q)}^{-}
\end{aligned}
$$

Xiao et al. (2014), estudou a remoção de $\mathrm{CO}_{2}$ a partir do biogás pelo sistema de lavagem com água. Foram analisados vários parâmetros, incluindo a razão líquido/gás, a pressão, a temperatura e o teor de $\mathrm{CO}_{2}$. Os resultados indicam que a taxa de remoção de $\mathrm{CO}_{2}$ aumentou de $34,6 \%$ a $94,2 \%$ quando a razão líquido/gás aumentou de 0,14 para 0,50. A taxa de remoção de $\mathrm{CO}_{2}$ pode chegar de $24,4 \%$ a $83,2 \%$ quando o teor de $\mathrm{CO}_{2}$ no gás era entre $25 \%$ e $45 \%$. Após a absorção, o menor teor de dióxido de carbono foi de 2,6\% em 1,2 MPa com vazões de $400{\mathrm{~L} . \mathrm{h}^{-1}}^{\text {para o }}$ gás e 200 L.h ${ }^{-1}$ para a água.

Schiavon Maia et al. (2015), avaliaram a purificação do biogás em batelada com um volume de $250 \mathrm{~mL}$ de Fe/EDTA, sintetizado em laboratório. Foram utilizadas concentrações de 0,02 e 0,04 mol. $\mathrm{L}^{-1}$ na coluna de absorção química com a passagem de $250 \mathrm{~mL} \cdot \mathrm{min}^{-1}$ de biogás. Os resultados de remoção de $\mathrm{H}_{2} \mathrm{~S}$ indicaram que a concentração da solução de Fe/EDTA exerce grande influência sobre a atividade catalítica. Foi possível remover completamente o $\mathrm{H}_{2} \mathrm{~S}$ do biogás em regime permanente utilizando uma razão $\mathrm{L} / \mathrm{G}$ de 0,46 , com um volume fixo de $250 \mathrm{~mL}$ de Fe/EDTA de concentração igual a $0,04 \mathrm{~mol} \cdot \mathrm{L}^{-1}$ na coluna de absorção química.

\section{MATERIAIS E MÉTODOS}

Segundo Horikawa et al. (2004), a solução de Fe/EDTA deve ser sintetizada em atmosfera inerte. Conforme descrito por Schiavon Maia et al. (2015), o tempo total para síntese desta solução pode levar, em média, até 10 horas.

Tendo em vista a grande dificuldade de síntese do Fe/EDTA em laboratório, avaliouse neste trabalho, a utilização de um complexo comercial que contém Fe/EDTA para a absorção química de $\mathrm{H}_{2} \mathrm{~S}$ do biogás.

Nos ensaios realizados foi utilizado um biogás sintético, cuja composição $\mathrm{mol} / \mathrm{mol}$ era igual a $2,2 \%$ de $\mathrm{H}_{2} \mathrm{~S}, 0,3 \%$ de $\mathrm{O}_{2}, 14 \%$ de $\mathrm{CO}_{2}, 2,4 \%$ de $\mathrm{N}_{2}$ e $81,1 \%$ de $\mathrm{CH}_{4}$. A fim de avaliar a remoção do $\mathrm{H}_{2} \mathrm{~S}$ e do $\mathrm{CO}_{2}$ do biogás 
foram realizados ensaios variando-se a concentração da solução de Fe/EDTA bem como a razão entre a vazão de líquido e gás (L/G) utilizando um sistema como o ilustrado na Figura 1.

Figura 1 - Diagrama da unidade experimental utilizada na purificação de biogás.

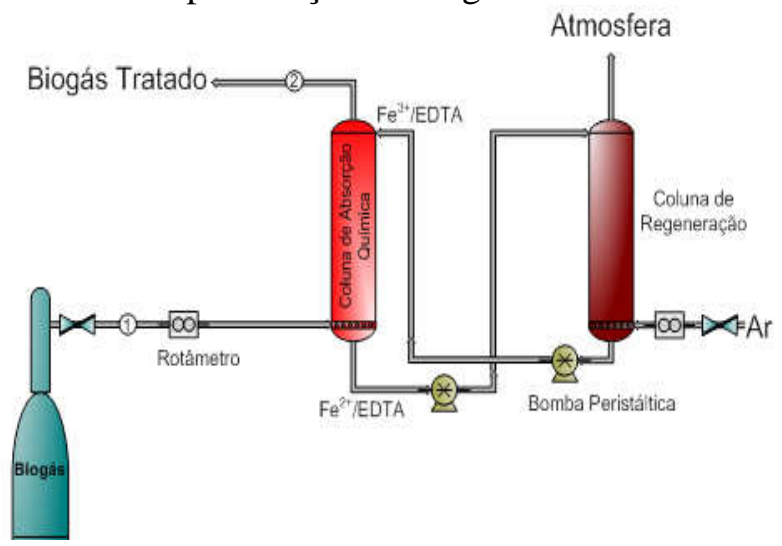

Os ensaios foram realizados à temperatura ambiente. A pressão na linha do biogás foi de $34,5 \mathrm{kPa}$, utilizando uma vazão fixa de biogás de $341 \mathrm{~mL} \cdot \mathrm{min}^{-1}$. O volume de líquido em cada coluna, de absorção química do $\mathrm{H}_{2} \mathrm{~S}$ e de regeneração da solução que contém Fe/EDTA, foi mantido constante em $250 \mathrm{~mL}$ para todos os experimentos. A composição do biogás foi medida por meio de Cromatografia Gasosa com Detector de Condutividade Térmica (GC/TCD) utilizando coluna capilar Plot U, com o auxílio do programa ChromQuest 4.1. Deste modo foi determinada a variação das composições de $\mathrm{H}_{2} \mathrm{~S}$ e $\mathrm{CO}_{2}$ nas amostras de biogás na alimentação e após a etapa de purificação.

O poder calorífico do biogás foi estimado segundo a metodologia descrita por Magalhães et al. (2004), levando em consideração que ele depende da porcentagem de metano existente no biogás e que o metano puro apresenta um poder calorífico de 9,9 kWh.m ${ }^{-3}$.

As variáveis estudadas no DCCR, em regime permanente, foram a concentração da solução comercial de Fe/EDTA e a razão L/G, nas quais os níveis utilizados são apresentados na Tabela 1.

Tabela 1 - Níveis das variáveis avaliadas no planejamento DCCR de acordo com a concentração de Fe/EDTA (C) e razão L/G.

\begin{tabular}{ccc}
\hline $\begin{array}{c}\text { Níveis das } \\
\text { Variáveis }\end{array}$ & $\begin{array}{c}\mathbf{C} \\
\left(\mathbf{m o l}^{\mathbf{2}} \mathbf{L}^{\mathbf{1}}\right)\end{array}$ & $\mathbf{L} / \mathbf{G}$ \\
\hline$-1,41$ & 0,1000 & 0,73 \\
-1 & 0,1145 & 0,81 \\
0 & 0,1500 & 1,00 \\
+1 & 0,1855 & 1,19 \\
$+1,41$ & 0,2000 & 1,27 \\
\hline
\end{tabular}

Na Tabela 2 é apresentada a matriz do planejamento experimental DCCR em termos de suas variáveis codificadas. As variáveis x 1 e $\mathrm{x} 2$ representam a codificação de $\mathrm{C}$ e $\mathrm{L} / \mathrm{G}$, respectivamente. As codificações foram realizadas utilizando-se as Equações 5 e 6.

$$
\begin{aligned}
& \mathrm{x} 1=(\mathrm{C}-0,15) / 0,0355 \\
& \mathrm{x} 2=(\mathrm{L} / \mathrm{G}-1) / 0,1915)
\end{aligned}
$$

Tabela 2 - Matriz do planejamento DCCR.

\begin{tabular}{cccc}
\hline Ensaios & & $\mathbf{x 1}$ & $\mathbf{x 2}$ \\
\hline 1 & & +1 & +1 \\
2 & Pontos fatoriais & +1 & -1 \\
3 & & -1 & +1 \\
4 & & -1 & -1 \\
\hline 5 & & 0 & 0 \\
6 & Pontos centrais & 0 & 0 \\
7 & & 0 & 0 \\
\hline 8 & & 0 & $+1,41$ \\
9 & \multirow{2}{*}{ Pontos axiais } & 0 & $-1,41$ \\
10 & & $+1,41$ & 0 \\
11 & & $-1,41$ & 0 \\
\hline
\end{tabular}

\section{RESULTADOS E DISCUSSÃO}

$\mathrm{Na}$ Tabela 3 são apresentadas as médias das eficiências de remoção de $\mathrm{H}_{2} \mathrm{~S}$ e $\mathrm{CO}_{2}$ para as condições estabelecidas no planejamento DCCR. 
Tabela 3 - Resultados para a eficiência de remoção de $\mathrm{H}_{2} \mathrm{~S}$ e $\mathrm{CO}_{2}$

\begin{tabular}{ccc}
\hline Ensaios & $\begin{array}{c}\text { Eficiência de } \\
\text { Remoção de } \\
\mathbf{H}_{2} \text { S (\%) }\end{array}$ & $\begin{array}{c}\text { Eficiência de } \\
\text { Remoção de } \\
\mathbf{C O}_{\mathbf{2}}(\boldsymbol{\%})\end{array}$ \\
\hline 1 & 64,90 & 28,06 \\
2 & 54,32 & 18,69 \\
3 & 59,68 & 26,88 \\
4 & 46,11 & 17,34 \\
5 & 64,90 & 26,18 \\
6 & 62,41 & 24,85 \\
7 & 60,88 & 28,39 \\
8 & 71,02 & 30,43 \\
9 & 55,95 & 16,80 \\
10 & 75,47 & 30,28 \\
11 & 58,63 & 22,44 \\
\hline
\end{tabular}

\subsection{Resultados para remoção de $\mathrm{H}_{2} \mathrm{~S}$}

Na Figura 2 é apresentado o Gráfico de Pareto relativo à remoção do $\mathrm{H}_{2} \mathrm{~S}$.

Figura 2 - Gráfico de Pareto para a Remoção de $\mathrm{H}_{2} \mathrm{~S}$.

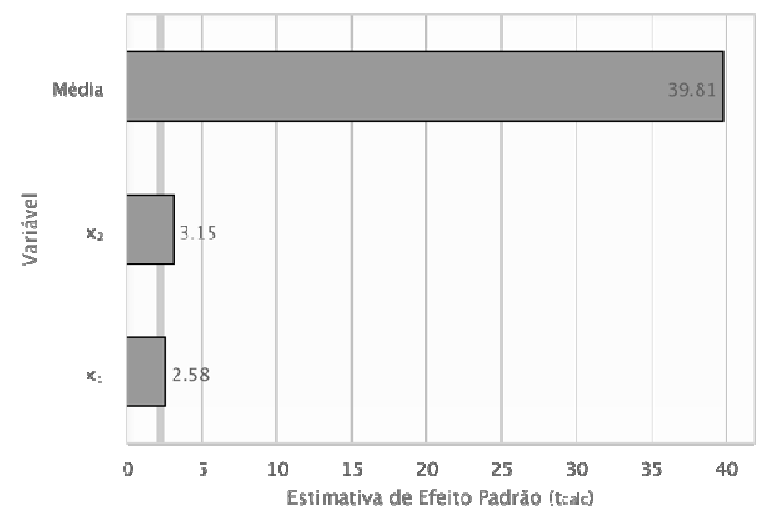

A partir da Figura 2 é possível verificar que tanto a concentração de Fe/EDTA (x1) quanto a razão $L / G(x 2)$ foram significativas, na remoção de $\mathrm{H}_{2} \mathrm{~S}$ do biogás, ao nível de $5 \%$. Estes parâmetros têm influência positiva sobre a purificação de biogás, indicando que caso haja um aumento no valor de $\mathrm{L} / \mathrm{G}$ e da concentração de Fe/EDTA a eficiência de remoção de $\mathrm{H}_{2} \mathrm{~S}$ tende a aumentar, confirmando os maiores resultados obtidos nos ensaios 8 e 10 apresentados na Tabela 3 .

$\mathrm{Na}$ Tabela 4 são apresentados os resultados da Análise de Variância (ANOVA), para a remoção de $\mathrm{H}_{2} \mathrm{~S}$ do biogás. Embora o modelo matemático, que representa os resultados de remoção seja válido $(p<0,05)$, o coeficiente de correlação $\left(R^{2}\right)$ apresentou um baixo valor $(67,42 \%)$, não sendo, entretanto, adequado para a predição de resultados. A equação 7 representa o modelo matemático obtido.

$\% \operatorname{RemH} 2 \mathrm{~S}=61,30+4,66 \cdot \mathrm{x} 1+5,68 \cdot \mathrm{x} 2$

A ausência de termos quadráticos significativos nesta equação indica que a região ótima encontra-se em um intervalo que não foi contemplado nestes experimentos.

Tabela 4 - Análise de Variância para o Planejamento DCCR na Remoção de $\mathrm{H}_{2} \mathrm{~S}$ do Biogás.

\begin{tabular}{lccccc}
\hline Fonte de Variação & $\begin{array}{c}\text { Soma dos } \\
\text { Quadrados }\end{array}$ & $\begin{array}{c}\text { Graus de } \\
\text { Liberdade }\end{array}$ & $\begin{array}{c}\text { Quadrado } \\
\text { Médio }\end{array}$ & F calc & p-valor \\
\hline Regressão & 431,8 & 2 & 215,9 & 8,3 & 0,01127 \\
Resíduos & 208,7 & 8 & 26,1 & & \\
Total & 640,4 & 10 & & & \\
\hline
\end{tabular}

Na Figura 3 são apresentadas as curvas de contorno para a eficiência de remoção de $\mathrm{H}_{2} \mathrm{~S}$. 
Figura 3 - Curvas de contorno para eficiência de remoção de $\mathrm{H}_{2} \mathrm{~S}$ em função das variáveis x1 e x2.

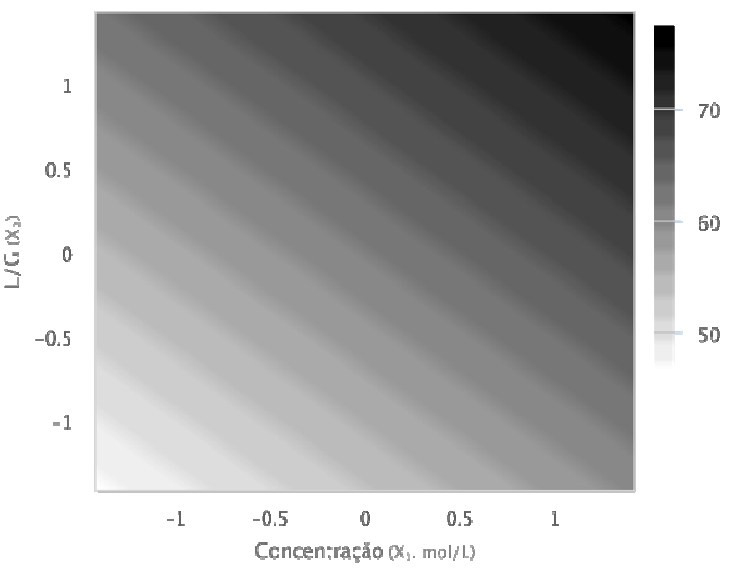

A partir da análise das curvas de contorno, geradas a partir do modelo matemático observadas na Figura 3 , é possível concluir que para valores elevados da razão L/G e de concentração de Fe/EDTA, maiores eficiências de remoção de $\mathrm{H}_{2} \mathrm{~S}$ são obtidas. Portanto, para alcançar uma remoção superior a 70\%, deve-se utilizar valores maiores do que 0,18 mol. $\mathrm{L}^{-1}$ e 1,19 para $\mathrm{C} \mathrm{e}$ $\mathrm{L} / \mathrm{G}$, respectivamente.

$\mathrm{Na}$ Figura 4 é possível observar a eficiência de remoção de $\mathrm{H}_{2} \mathrm{~S}$ ao longo do tempo para os ensaios experimentais 1, 2, 3 e 4 com a solução comercial que contém Fe/EDTA.

Figura 4 - Eficiência de remoção de $\mathrm{H}_{2} \mathrm{~S}$ com a solução comercial para os ensaios 1, 2, 3 e 4 .

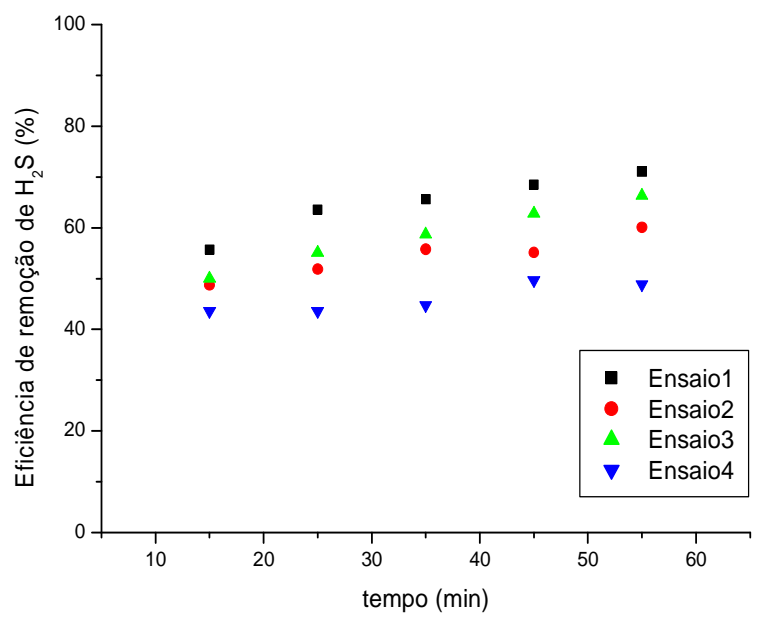

De acordo com a Figura 4, o ensaio 1 com maior $\mathrm{L} / \mathrm{G}$ e maior concentração de Fe/EDTA $\quad(0,1855 \quad \mathrm{~mol} / \mathrm{L}$ e $\mathrm{L} / \mathrm{G} \quad 1,19)$ apresentou maior eficiência de remoção de $\mathrm{H}_{2} \mathrm{~S}$ ao longo do tempo, chegando a $71 \%$ no tempo de $55 \mathrm{~min}$, confirmando o previsto nas curvas de contorno. De um modo geral, houve um aumento na eficiência com o tempo para os quatro ensaios, possivelmente devido à formação de partículas de enxofre no meio reacional, aumentando a resistência à passagem de biogás pela solução comercial, havendo maior contato entre gás e líquido, como se fosse uma coluna recheada.

As eficiências de remoção de $\mathrm{H}_{2} \mathrm{~S}$, com a solução comercial que contém Fe/EDTA, para os ensaios 5, 6 e 7 estão apresentadas na Figura 5.

Figura 5 - Eficiência de remoção de $\mathrm{H}_{2} \mathrm{~S}$ com a solução comercial para os ensaios 5, 6 e 7 .

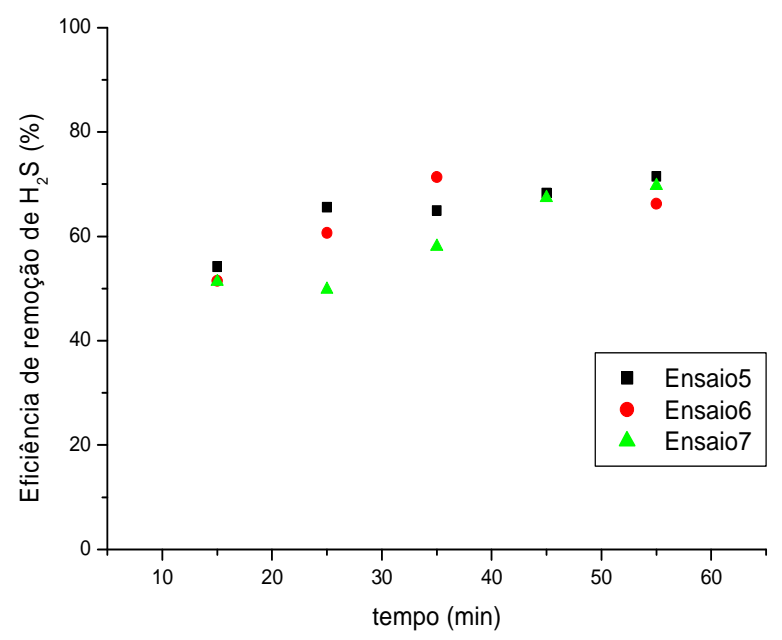

Os ensaios 5, 6 e 7, ambos na concentração de $0,1500 \mathrm{~mol} / \mathrm{L}$ de Fe/EDTA e L/G 1,00 (ponto central do DCCR) apresentaram uma eficiência média de absorção de $\mathrm{H}_{2} \mathrm{~S}$ de $63 \%$.

$\mathrm{Na}$ Figura 6 estão apresentadas as eficiências de remoção de $\mathrm{H}_{2} \mathrm{~S}$ com a solução comercial para os ensaios 8, 9, 10 e 11 . 
Figura 6 - Eficiência de remoção de $\mathrm{H}_{2} \mathrm{~S}$ com a solução comercial para os ensaios 8, 9, 10 e 11.

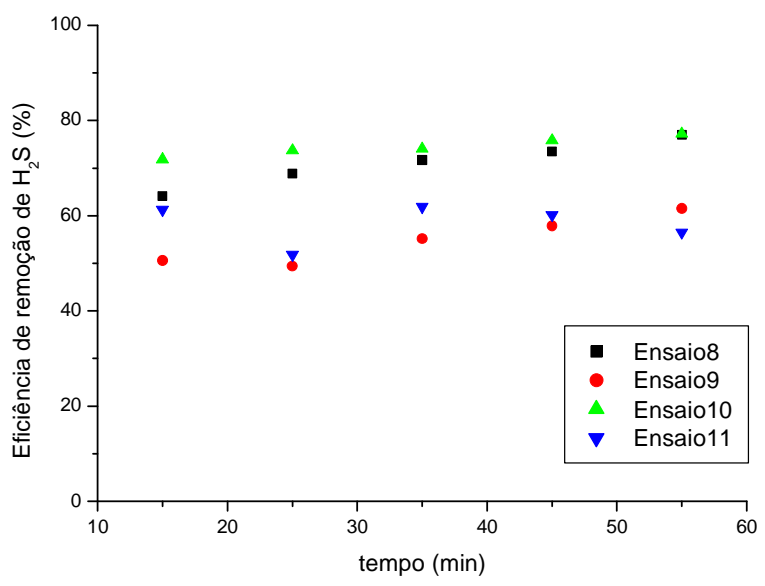

Comparando os ensaios 8 e 9, com mesma concentração de Fe/EDTA, a eficiência de absorção de $\mathrm{H}_{2} \mathrm{~S}$ do ensaio 8, com maior $\mathrm{L} / \mathrm{G}$, foi superior ao ensaio 9, conforme mostra a Figura 6. O ensaio 10, com o dobro da concentração do ensaio $11 \mathrm{e}$ mesmo L/G, apresentou em média $75 \%$ de eficiência de absorção de $\mathrm{H}_{2} \mathrm{~S}$ ao longo do tempo, enquanto o ensaio 11 apresentou em média $59 \%$.

\subsection{Resultados para a remoção de $\mathrm{CO}_{2}$}

$\mathrm{Na}$ Figura 7 é apresentado o Gráfico de Pareto relativo à remoção do $\mathrm{CO}_{2}$.

Figura 7 - Gráfico de Pareto para a remoção de $\mathrm{CO}_{2}$.

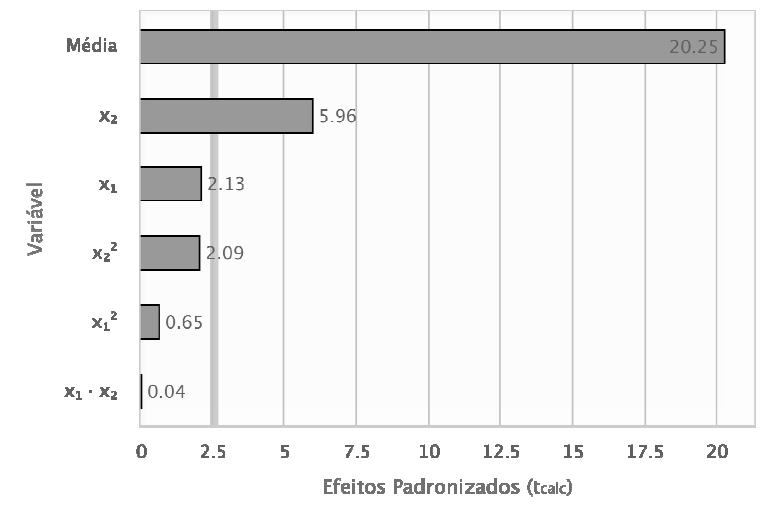

De acordo com o Gráfico de Pareto, apresentado na Figura 7, a razão L/G (x2) é a única variável significativa ao nível de $5 \%$ que possui influência na absorção do $\mathrm{CO}_{2}$ do biogás. A variável $\mathrm{C} \quad(\mathrm{x} 1)$ não foi significativa, ou seja, o aumento da concentração de Fe/EDTA não gera um aumento da eficiência de remoção de $\mathrm{CO}_{2}$. Desta forma, pode-se trabalhar em baixos valores de $\mathrm{C}$ durante os experimentos.

Portanto, se houver um aumento no valor de $\mathrm{L} / \mathrm{G}$, a eficiência de remoção de $\mathrm{CO}_{2}$ tende a aumentar, como mostra o resultado obtido no ensaio 8 da Tabela 3.

Na Figura 8 são apresentadas as curvas de contorno para eficiência de remoção de $\mathrm{CO}_{2}$.

Figura 8 - Curvas de contorno para eficiência de remoção de $\mathrm{CO}_{2}$ em função das variáveis x1 e x2 .

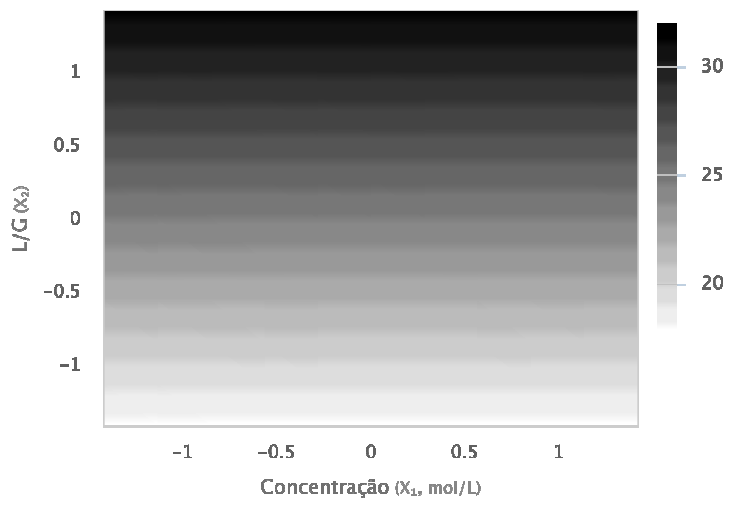

Observa-se nas curvas de contorno da Figura 8 que, nos intervalos investigados, as maiores eficiências de remoção ocorreram para níveis altos de $L / G$ sem influência significativa da concentração de Fe/EDTA.

$\mathrm{Na}$ Tabela 5 são apresentados os resultados da análise de variância do modelo matemático para a remoção de $\mathrm{CO}_{2}$ do biogás. $\mathrm{O}$ modelo matemático, que representa os resultados de remoção, mostrou-se válido $(\mathrm{p}<0,05)$, com um coeficiente de correlação de $71,91 \%$. A equação 8 representa o modelo matemático obtido.

$\% \operatorname{RemCO} 2=24,54+4,77 \times \mathrm{x} 2$ 
Tabela 5 - Análise de Variância Obtida para o Planejamento DCCR na Remoção de $\mathrm{CO}_{2}$ do Biogás.

\begin{tabular}{lccccc}
\hline $\begin{array}{l}\text { Fonte de } \\
\text { Variação }\end{array}$ & $\begin{array}{c}\text { Soma dos } \\
\text { Quadrados }\end{array}$ & $\begin{array}{c}\text { Graus de } \\
\text { Liberdade }\end{array}$ & $\begin{array}{c}\text { Quadrado } \\
\text { Médio }\end{array}$ & Fcalc & p-valor \\
\hline Regressão & 182,3 & 1 & 182,3 & 23,0 & 0,00097 \\
Resíduos & 71,2 & 9 & 7,9 & & \\
Total & 253,5 & 10 & & & \\
\hline
\end{tabular}

$\mathrm{Na}$ Figura 9 pode-se observar a eficiência de remoção de $\mathrm{CO}_{2}$ em função de $\mathrm{C}$ e L/G para os ensaios 1, 2, 3 e 4.

Figura 9 - Eficiência de remoção de $\mathrm{CO}_{2}$ com a solução comercial para os ensaios $1,2,3$ e 4 .

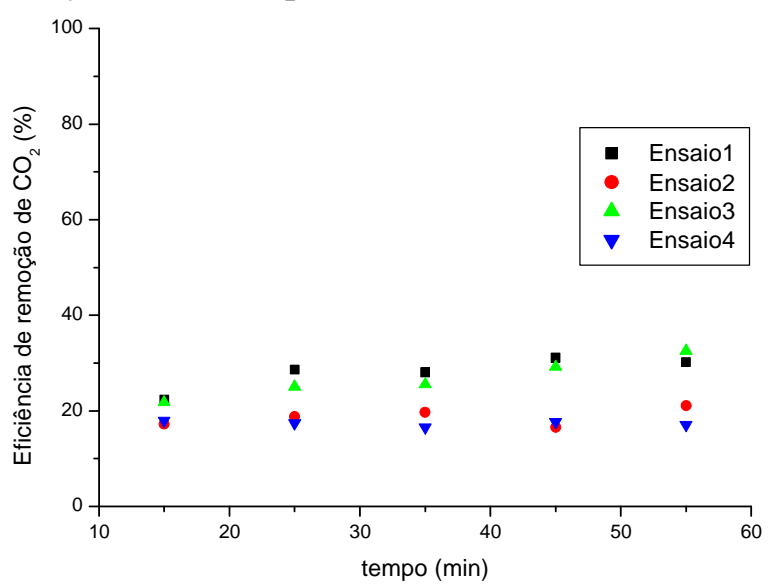

De acordo com o observado na Figura 9, os ensaios 1 e 3 , com L/G $(1,19)$ maior que os ensaios 2 e 4, apresentaram maior eficiência de absorção de $\mathrm{CO}_{2}$ ao longo do tempo, porém a concentração da solução não apresentou significância na retirada do $\mathrm{CO}_{2}$, conforme previsto nas curvas de contorno da Figura 8.

Na Figura 10 tem-se os resultados para as eficiências de remoção de $\mathrm{CO}_{2}$ com a solução comercial para os ensaios 5, 6 e 7 .
Figura 10 - Eficiência de remoção de $\mathrm{CO}_{2}$ com a solução comercial para os ensaios 5, 6 e 7.

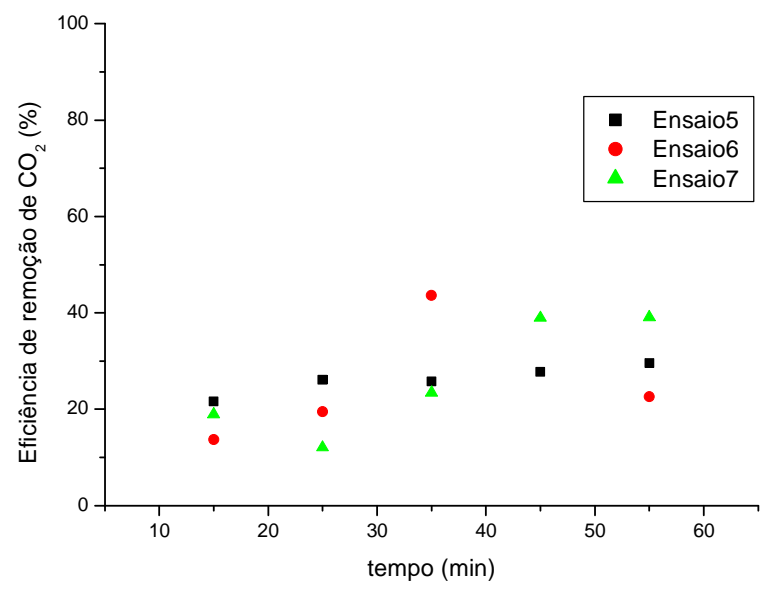

De acordo com a Figura 10, os ensaios 5,6 e 7, ambos na concentração de 0,1500 mol/L de Fe/EDTA e L/G 1,00, apresentaram uma eficiência média de absorção de $\mathrm{CO}_{2}$ de $26 \%$. Os ensaios $8,9,10$ e 11 estão apresentados na Figura 11.

Figura 11 - Eficiência de remoção de $\mathrm{CO}_{2}$ com a solução comercial para os ensaios 8, 9, 10 e 11 .

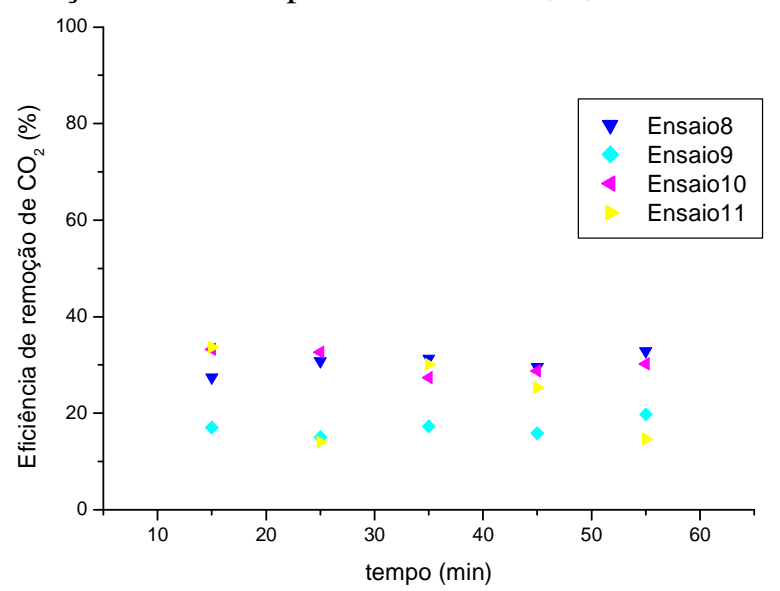

Os ensaios 8 e 10 foram mais eficientes na absorção física de $\mathrm{CO}_{2}$, sendo que o ensaio 
8 possuía maior $\mathrm{L} / \mathrm{G}(1,27)$ e o ensaio 10 maior concentração de Fe/EDTA $(2,0000 \mathrm{~mol} / \mathrm{L})$.

Como a composição de entrada na unidade experimental era de $14 \% \mathrm{~mol} / \mathrm{mol}$ de $\mathrm{CO}_{2}$, foi possível alcançar uma eficiência máxima de absorção de $\mathrm{CO}_{2}$ de $30,43 \%$ (ensaio 8) do biogás de entrada na unidade experimental. Isso configura um acréscimo de $5,25 \%$ no poder calorífico do biogás.

\section{CONCLUSÕES}

A solução comercial que contém Fe/EDTA apresentou boa viabilidade para a remoção de $\mathrm{H}_{2} \mathrm{~S}$ do biogás. Nos intervalos investigados, os valores elevados da razão L/G e de concentração de Fe/EDTA levam a maiores eficiências de remoção de $\mathrm{H}_{2} \mathrm{~S}$. Para o $\mathrm{CO}_{2}$, a melhor remoção ocorreu em níveis altos de L/G sem influência significativa da concentração de Fe/EDTA. O aumento do poder calorífico do biogás, pela remoção do $\mathrm{CO}_{2}$, foi de $8,03 \mathrm{kWh} / \mathrm{m}^{3}$ para $8,45 \mathrm{kWh} / \mathrm{m}^{3}$, o que representa um ganho de $5,25 \%$.

\section{NOMENCLATURA}

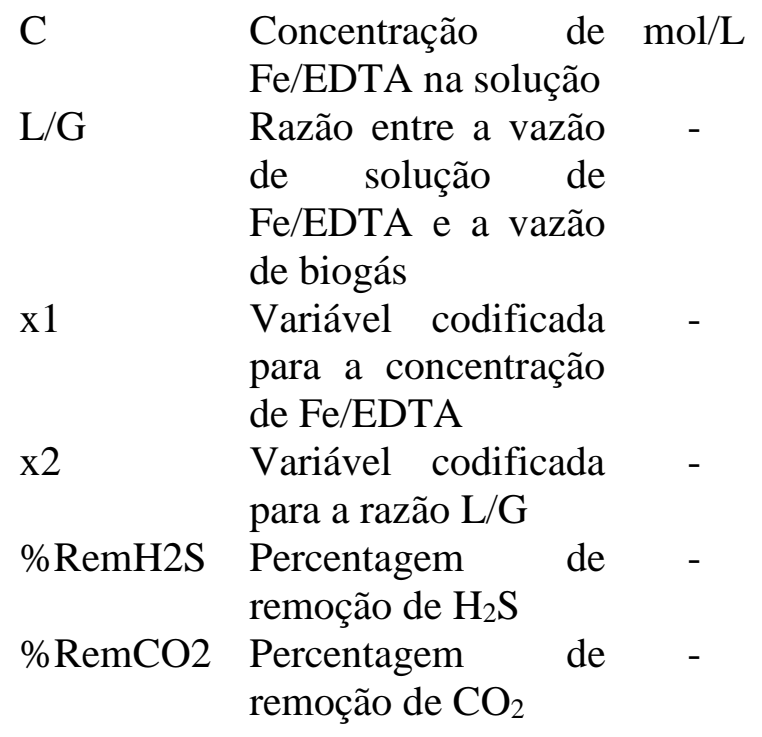

\section{REFERÊNCIAS}

HINTON, N.; STONE, R. Laminar burning velocity measurements of methane and carbon dioxide mixtures (biogas) over wide ranging temperatures and pressures. Fuel, v. 116, Pg 743-750, 2014.

HORIKAWA, M.S.; ROSSI, F.; GIMENES, M.L.; COSTA, C.M.M.; SILVA, M.G.C. Chemical Absorption of $\mathrm{H}_{2} \mathrm{~S}$ for Biogas Purification. Brazilian Journal of Chemical Engineering, v. 21, v. 03, pp. 415 - 422, 2004.

KÁRÁSZOVÁ, M.; VEJRAŽKA, J.; VESELÝ, V.; FRIESS, K.; RANDOVÁ, A.; HEJTMÁNEK, V.; BRABEC, L.; IZÁK, P. A water-swollen thin film composite membrane for effective upgrading of raw biogas by methane, Separation and Purification Technology, v. 89, 22, p. 212-216, 2012.

MAAT, H. ter; HOGENDOORNB, J. A.; VERSTEEG, G. F. The removal of hydrogen sulfide from gas streams using an aqueous metal sulfate absorbent Part I. The absorption of hydrogen sulfide in metal sulfate solutions, Separation and Purification Technology, 43. 183-197, 2005.

MAGALHÃES, E. A.; SOUZA, S. N. M.; AFONSO, A. D. L.; RICIERI, R. P. Confecção e avaliação de um sistema de remoção do $\mathrm{CO} 2$ contido no biogás. Acta Scientiarum Technology, v. 26, n. 1, p. 1119, 2004.

NUTIU, E. Anaerobic Purification Installation with Production of Biogas and Liquid Fertilizers, Procedia Technology, v. 12, p. 632-636, 2014.

RYCKEBOSCH， E.; DROUILLON， M.; VERVAEREN, $H$. Techniques for transformation of biogas to biomethane, 
Biomass and Bioenergy, Volume 35, Issue 5, May 2011, Pages 1633-1645, 2011.

SCHIAVON MAIA, D. C.; LENZI, G. G.; ARROYO, P. A.; FRARE, L. M.; GIMENES, M. L.; PEREIRA, N. C. Desenvolvimento de um Sistema para Purificação de Biogás Utilizando Fe/EDTA como Absorvente. Engevista, v. 17, n. 2, p. 219-231, 2015.

SIGOT, L.; DUCOM, G.; BENADDA, B.; LABOURÉ, C. Adsorption of octamethylcyclotetrasiloxane on silica gel for biogas purification, Fuel, v. 135, p. 205-209, 2014.

XIAO, Y.; YUAN, H.; PANG, Y.; CHEN, S.; ZHU, B.; ZOU, D.; MA, J.; YU, L.; LI, X. $\mathrm{CO}_{2}$ Removal from Biogas by Water Washing System, Chinese Journal of Chemical Engineering, v. 22, Issue 8, p. 950-953, 2014.

WUBS, H. J.; BEENACKERS, A. A. C. M. Kinetics of the Oxidation of Ferrous Chelates of EDTA and HEDTA in Aqueous Solution. Ind. Eng. Chem. Res., 32, p. 2580 - 2594, 1993. 